\title{
Effects of asenapine on agitation and hostility in adults with acute manic or mixed episodes associated with bipolar I disorder
}

This article was published in the following Dove Press journal: Neuropsychiatric Disease and Treatment

\author{
Leslie Citrome' \\ Ronald Landbloom ${ }^{2}$ \\ Cheng-Tao Chang ${ }^{3}$ \\ Willie Earley ${ }^{4}$ \\ 'Department of Psychiatry and \\ Behavioral Sciences, New York \\ Medical College, Valhalla, NY, USA; \\ ${ }^{2}$ Department of Neuroscience, \\ Merck, Whitehouse Station, NJ, USA; \\ ${ }^{3}$ Biostatistics, Allergan, Jersey City, NJ, \\ USA; ${ }^{4}$ Clinical Development, Allergan, \\ Jersey City, NJ, USA
}

Background: Bipolar disorder is associated with an increased risk of aggression. However, effective management of hostility and/or agitation symptoms may prevent patients from becoming violent. This analysis investigated the efficacy of the antipsychotic asenapine on hostility and agitation in patients with bipolar I disorder.

Methods: Data were pooled from three randomized, double-blind, placebo-controlled, Phase III trials of asenapine in adults with manic or mixed episodes of bipolar I disorder (NCT00159744, NCT00159796, and NCT00764478). Post hoc analyses assessed the changes from baseline to day 21 on the Young Mania Rating Scale (YMRS) and the Positive and Negative Syndrome Scale (PANSS) hostility-related item scores in asenapine- or placebo-treated patients with at least minimal or mild symptom severity and on the PANSS-excited component (PANSS-EC) total score in agitated patients. Changes were adjusted for improvements in overall mania symptoms to investigate direct effects on hostility.

Results: Significantly greater changes in favor of asenapine versus placebo were observed in YMRS hostility-related item scores (irritability: least squares mean difference [ $95 \%$ confidence interval $]=-0.5[-0.87,-0.22], P=0.001$; disruptive-aggressive behavior: $-0.7[-0.99,-0.37]$, $P<0.0001)$, PANSS hostility item score $(-0.2[-0.44,-0.04] ; P=0.0181)$, and PANSS-EC total score $(-1.4[-2.4,-0.4] ; P=0.0055)$. Changes in the YMRS disruptive-aggressive behavior score and the sum of the hostility-related items remained significant after adjusting for improvements in other YMRS item scores.

Conclusion: Asenapine significantly reduced hostility and agitation in patients with bipolar I disorder; improvement was at least partially independent of overall improvement on mania symptoms.

Keywords: asenapine, hostility, bipolar disorder, agitation, mania, aggression

\section{Introduction}

Violent/aggressive behavior in psychiatric patients is a public health problem associated with significant medical, emotional, and financial costs to patients, caregivers, and society. ${ }^{1}$ Aggressive behavior, defined as overt action intended to harm, ${ }^{1}$ is frequently observed in patients with bipolar disorder, particularly in those with comorbid substance abuse. ${ }^{1-4}$ In patients with bipolar disorder, aggressive behavior has been linked to comorbid personality disorder, history of childhood trauma, and the severity of current manic or depressive symptoms. ${ }^{5}$ Because patients with bipolar disorder are at high risk of becoming violent during acute manic episodes, ${ }^{6,7}$ treatment of at-risk patients before their symptoms progress to violence is critical.
Correspondence: Leslie Citrome II Medical Park Drive, Suite 106, Pomona, NY 10970, USA

Tel + I 9146722692

Fax +l 8453628745

Email citrome@cnsconsultant.com 
Behaviors such as hostility and agitation can be used to identify patients who are at risk of aggressive behavior. Hostility denotes unfriendly attitudes, including irritability, anger, resentment, and verbal aggression. ${ }^{1}$ Although not violent behavior per se, hostility can escalate into violence and is correlated with an increased risk of aggression and treatment nonadherence in patients with psychiatric disorders. ${ }^{1,8}$ Similarly, agitation, which is defined as excessive verbal or motor activity, can also escalate to aggressive behavior ${ }^{9}$ and is frequently observed in patients with bipolar disorder who are experiencing a manic episode. ${ }^{1}$ Due to the risk of hostility and agitation leading to violent behavior, effective management of these conditions is necessary to ensure patient safety and to prevent harm to others.

Current treatments to manage hostility and agitation in patients with bipolar disorder who are experiencing a manic episode include benzodiazepines and antipsychotics. ${ }^{1,10}$ Post hoc analyses of data from the Clinical Antipsychotic Trials of Intervention Effectiveness (CATIE) study in patients with schizophrenia suggested that some second-generation antipsychotics may have specific effects on hostility, independent of their effects on positive schizophrenia symptoms. ${ }^{11}$ Importantly, some of these antipsychotics have been shown to have similar effects on hostility and agitation in patients with bipolar disorder. ${ }^{12-14}$

The second-generation antipsychotic asenapine is US Food and Drug Administration approved in the USA for acute and maintenance treatment of schizophrenia and for acute and maintenance treatment of bipolar I disorder in adults. Asenapine monotherapy has demonstrated efficacy in three positive, randomized, double-blind, placebo-controlled, Phase III trials in adults with manic or mixed episodes associated with bipolar I disorder. ${ }^{15-17}$ Unlike other antipsychotics, asenapine is administered as a fast-dissolving sublingual tablet twice daily (BID). In a randomized, double-blind, placebocontrolled trial in patients with any diagnosis experiencing acute agitation in an emergency room setting, sublingual asenapine demonstrated efficacy in treating agitation, with an effect size (ES) comparable to that observed in a prior study of intramuscular antipsychotics, ${ }^{18}$ suggesting that it may have efficacy as a noninvasive method of managing hostile and agitated behaviors in patients with bipolar disorder. To better characterize the effects of asenapine on hostility and agitation in patients experiencing a manic or mixed episode associated with bipolar I disorder, post hoc analyses based on Young Mania Rating Scale (YMRS) ${ }^{19}$ and Positive and Negative Syndrome Scale (PANSS) ${ }^{20}$ items related to hostility and agitation were conducted on pooled data from the three double-blind, randomized studies.

\section{Methods}

\section{Study design}

Data were pooled from three similarly designed, 3-week, multicenter, positive, randomized, double-blind, placebocontrolled, Phase III trials of asenapine in adult patients with manic or mixed episodes associated with bipolar I disorder (NCT00159744, NCT00159796, and NCT00764478). The data accessed from these trials are on file with the study sponsor. Detailed methods of the component studies have been previously published. ${ }^{15-17}$ In brief, trials included a run-in period of up to 7 days and 3 weeks of double-blind treatment. Two studies used flexible-dose designs in which patients were randomized 2:1:2 to asenapine, placebo, and olanzapine (included for assay sensitivity). The starting asenapine dose was $10 \mathrm{mg}$ BID on day 1; thereafter, patients received 5 or $10 \mathrm{mg}$ BID. The third study used a fixed-dose design, and patients were randomized 1:1:1 to placebo, asenapine $5 \mathrm{mg}$ BID, or asenapine $10 \mathrm{mg}$ BID. Each component study was approved by an institutional review board and conducted in accordance with the Declaration of Helsinki and Good Clinical Practice guidelines. All participants provided written informed consent.

\section{Patients}

The studies included male and female patients ( $\geq 18$ years old) with a primary diagnosis of bipolar I disorder according to Diagnostic and Statistical Manual of Mental Disorders, fourth edition (DSM-IV) ${ }^{21}$ criteria who were experiencing a manic or mixed episode. The Mini-International Neuropsychiatric Interview ${ }^{22}$ was used to confirm the diagnosis; mania severity was determined using the YMRS. Clinical inclusion criteria included YMRS total score $\geq 20$ at screening and baseline, a current manic or mixed bipolar I episode that began $\leq 3$ months (flexible-dose studies) or $\leq 1$ month (fixed-dose study) prior to screening, and a documented history of $\geq 1$ previous moderate-to-severe manic or mixed episodes, with or without psychotic features.

Typical exclusion criteria for bipolar I disorder studies were applied. Patients with any other psychotic disorders, primary axis I diagnosis other than bipolar I disorder, current diagnosis of substance abuse/dependence, history of rapid cycling, and who were at imminent risk of harm to self or others were excluded. Use of antipsychotics, depot neuroleptics, antidepressants, mood stabilizers, and other psychotropic drugs was prohibited; drugs for extrapyramidal symptoms (eg, beta-blockers and anticholinergics), agitation (eg, lorazepam and diazepam), and insomnia (eg, temazepam, zolpidem, and zaleplon) were allowed during screening and for the first 7 days after randomization only. 


\section{Post hoc analyses}

Analyses were based on pooled data from the three component studies. The full analysis set (FAS) comprised data from all randomized patients who received at least one dose of trial medication and had both baseline and $\geq 1$ postbaseline YMRS total score assessments. Asenapine doses (5 or $10 \mathrm{mg}$ BID) were combined for the pooled analysis. To evaluate the effects of asenapine on hostility in patients with a manic or mixed episode associated with bipolar I disorder, post hoc analyses were performed in subgroups of patients with hostility symptoms defined by cutoff scores on hostility-related items from the YMRS or PANSS. The YMRS hostility subgroup included patients with baseline scores $\geq 2$ on the YMRS hostility-related items (ie, disruptive-aggressive behavior and irritability); the PANSS hostility subgroup included patients with baseline scores $\geq 2$ on the PANSS hostility item (P7). YMRS total scores and scores on individual items were assessed in both the YMRS and PANSS hostility subgroups. PANSS hostility item scores were assessed in the PANSS hostility subgroup.

To characterize the effects of asenapine on agitation, changes from baseline in PANSS-excited component (PANSS-EC) total scores were evaluated in agitated patients treated with asenapine or placebo. The PANSS-EC comprises the following five PANSS items: tension (G4), uncooperativeness (G8), poor impulse control (G14), excitement (P4), and hostility (P7). ${ }^{23}$ PANSS-EC agitation subgroup patients were defined as those having a PANSS-EC total score $\geq 14$ and one or more individual PANSS-EC item scores $\geq 4$ at baseline. The percentage of patients who achieved response (ie, $\geq 40 \%$ decrease from baseline to endpoint) in PANSS-EC total score was also determined.

\section{Statistical analyses}

Least squares (LS) mean changes from baseline to day 21 in YMRS total score and individual item scores were assessed for asenapine- and placebo-treated patients in the YMRS and PANSS hostility subgroups. LS mean change in the PANSS hostility item score was assessed in the PANSS hostility subgroup; LS mean change in the PANSS-EC total score was assessed in the PANSS-EC agitation subgroup. LS mean differences (LSMDs) with 95\% confidence intervals (CIs) for asenapine versus placebo were used to evaluate changes from baseline in efficacy parameters. Analyses were based on a mixed-effects model for repeated measures (MMRM) approach with an unstructured covariance matrix with baseline, pooled site, treatment, and visit-by-treatment interaction as fixed effects. LS means at day 21 were estimated using this MMRM model, and including early visit data based on the assumption of missing at random. For the PANSS-EC responder analysis, a logistic regression model with study and treatment as factors and baseline PANSS-EC score as a covariate was used, with missing values imputed based on a multiple imputation method.

To distinguish between specific improvements in hostility symptoms and general improvements in overall mania symptoms, separate analyses were conducted using a similar MMRM model with additional covariates to correct for potential confounder variables. On the YMRS disruptive-aggressive behavior score, the irritability item score, and the sum of the disruptive-aggressive behavior and irritability item scores, analyses were conducted after adjusting for the sum changes of the other nine individual YMRS items. Similarly, analyses were conducted on the PANSS hostility item scores after adjusting for scores on the PANSS positive symptom items (delusions [P1], conceptual disorganization [P2], hallucinatory behavior [P3], grandiosity [P5], suspiciousness/persecution [P6], and unusual thought content [G9]). Additional analyses were conducted after adjusting for the PANSS positive symptom items and sedation (as measured by the presence of an adverse event of sedation, somnolence, or hypersomnia) as covariates.

ESs were calculated using Cohen's $d$ and were based on the difference between treatment group means divided by the pooled standard deviation from the two treatment groups. All tests for LSMD were two sided at the 5\% significance level; $P$-values were not adjusted for multiple comparisons.

\section{Results \\ Pooled baseline characteristics}

Data from 929 patients were included in the pooled FAS (pooled asenapine $=605$ patients; placebo $=324$ patients). The YMRS hostility subgroup included data from 741 patients at baseline (pooled asenapine $=481$; placebo $=260$ ). The PANSS hostility subgroup included data from 693 patients at baseline (pooled asenapine $=455$ patients; placebo $=238$ patients), and the PANSS-EC agitation subgroup included 349 patients (pooled asenapine $=233$ patients; placebo $=116$ patients). Baseline scores on YMRS or PANSS items/factors related to agitation or hostility were similar between treatment groups (Table 1). Among all patients in the component trials, the most commonly used concomitant medications for agitation were lorazepam (placebo, 26.7\%; asenapine, 24.0\%) and diazepam (placebo, 1.5\%; asenapine, $1.0 \%$ ); no other individual concomitant medications were administered for agitation in $>0.5 \%$ of patients. 
Table I Baseline scores on efficacy scales and items related to hostility and agitation

\begin{tabular}{|c|c|c|c|c|}
\hline Efficacy scale & $\begin{array}{l}\text { Placebo } \\
\text { patients }(n)\end{array}$ & Placebo, mean (SD) & $\begin{array}{l}\text { Asenapine } \\
\text { patients (n) }\end{array}$ & Asenapine, mean (SD) \\
\hline \multicolumn{5}{|l|}{ YMRS hostility subgroup ${ }^{a}$} \\
\hline YMRS total score & 260 & $30.2(5.94)$ & 481 & $30.3(5.97)$ \\
\hline YMRS disruptive-aggressive behavior item score & 260 & $3.3(I .31)$ & 481 & $3.3(1.22)$ \\
\hline YMRS irritability item score & 260 & $4.1(1.14)$ & 481 & $4.1(1.20)$ \\
\hline PANSS total score & 259 & $64.5(17.37)$ & 476 & $62.8(15.4 I)$ \\
\hline PANSS positive subscale score & 259 & $18.2(5.74)$ & 476 & $17.8(5.24)$ \\
\hline PANSS hostility item (P7) score & 259 & $2.7(1.19)$ & 476 & $2.7(1.18)$ \\
\hline PANSS-EC score & 259 & $13.6(3.72)$ & 476 & $13.6(3.75)$ \\
\hline \multicolumn{5}{|l|}{ PANSS hostility subgroup ${ }^{b}$} \\
\hline YMRS total score & 236 & $29.8(6.02)$ & 452 & $29.9(6.08)$ \\
\hline YMRS disruptive-aggressive behavior item score & 236 & $3 . \mathrm{I}(\mathrm{I} .5 \mathrm{I})$ & 452 & $3.1(1.45)$ \\
\hline YMRS irritability item score & 236 & $4.0(1.30)$ & 452 & $4.0(1.29)$ \\
\hline PANSS total score & 238 & $66.5(17.04)$ & 455 & $64.7(15.12)$ \\
\hline PANSS positive subscale score & 238 & $18.9(5.54)$ & 455 & $18.5(4.96)$ \\
\hline PANSS hostility item (P7) score & 238 & $3.1(0.93)$ & 455 & $3.0(0.94)$ \\
\hline PANSS-EC score & 238 & $14.2(3.31)$ & 455 & $14.1(3.43)$ \\
\hline \multicolumn{5}{|l|}{ PANSS-EC agitation subgroup ${ }^{c}$} \\
\hline YMRS total score & 115 & $31.9(6.36)$ & 232 & $32.4(6.21)$ \\
\hline YMRS disruptive-aggressive behavior item score & 115 & $3.6(1.48)$ & 232 & $3.5(1.43)$ \\
\hline YMRS irritability item score & 115 & $4.5(1.29)$ & 232 & $4.4(1.36)$ \\
\hline PANSS total score & 116 & $75.0(17.04)$ & 233 & $71.2(14.84)$ \\
\hline PANSS positive subscale score & 116 & $21.8(5.77)$ & 233 & $20.8(5.19)$ \\
\hline PANSS hostility item (P7) score & 116 & $3.4(1.14)$ & 233 & $3.4(1.15)$ \\
\hline PANSS-EC score & 116 & $16.8(2.67)$ & 233 & $16.7(2.60)$ \\
\hline
\end{tabular}

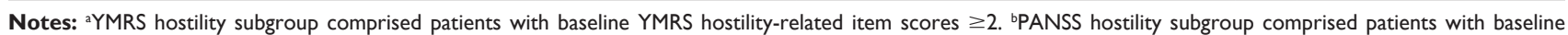
PANSS hostility item scores $\geq 2$. 'PANSS-EC agitation subgroup comprised patients with PANSS-EC score $\geq 14$, and one or more individual PANSS-EC item scores $\geq 4$ at baseline.

Abbreviations: PANSS, Positive and Negative Syndrome Scale; PANSS-EC, PANSS-excited component; SD, standard deviation; YMRS, Young Mania Rating Scale.

\section{YMRS hostility subgroup}

For patients in the YMRS hostility subgroup, baseline YMRS total score and baseline scores on all 11 YMRS items were similar between treatment groups (Table 2). A significantly greater change from baseline to day 21 on YMRS total score was observed with asenapine versus placebo $(\mathrm{ES}=0.45$, $P<0.0001$ ) (Table 2). Significantly larger changes from baseline in favor of asenapine were also observed on both YMRS

Table 2 Change from baseline to day 21 in YMRS total score and individual items in the YMRS hostility subgroup ${ }^{\mathrm{a}}$ (MMRM)

\begin{tabular}{|c|c|c|c|c|c|c|c|}
\hline & \multicolumn{2}{|c|}{ Placebo $(n=260)$} & \multicolumn{2}{|c|}{ Asenapine $(n=48 I)$} & \multicolumn{3}{|c|}{ Asenapine vs placebo } \\
\hline & $\begin{array}{l}\text { Baseline } \\
\text { mean (SD) }\end{array}$ & $\begin{array}{l}\text { LS mean } \\
\text { change (SE) }\end{array}$ & $\begin{array}{l}\text { Baseline } \\
\text { mean (SD) }\end{array}$ & $\begin{array}{l}\text { LS mean } \\
\text { change (SE) }\end{array}$ & $\begin{array}{l}\text { LSMD } \\
(95 \% \mathrm{CI})\end{array}$ & ES & $P$-value \\
\hline YMRS total score & $30.2(5.94)$ & $-9.7(0.76)$ & $30.3(5.97)$ & $-14.0(0.55)$ & $-4.3(-6.16,-2.54)$ & 0.45 & $<0.0001$ \\
\hline \multicolumn{8}{|l|}{ YMRS items } \\
\hline Disruptive-aggressive behavior & $3.3(\mathrm{I} .3 \mathrm{I})$ & $-0.9(0.13)$ & $3.3(1.22)$ & $-1.5(0.09)$ & $-0.7(-0.99,-0.37)$ & 0.45 & $<0.000$ I \\
\hline Irritability & $4.1(1.14)$ & $-1.3(0.14)$ & $4.1(1.20)$ & $-1.9(0.10)$ & $-0.5(-0.87,-0.22)$ & 0.32 & 0.0010 \\
\hline Appearance & $1.2(0.84)$ & $-0.4(0.06)$ & $1.2(0.90)$ & $-0.6(0.04)$ & $-0.2(-0.30,-0.01)$ & 0.21 & 0.0329 \\
\hline Insight & $1.0(1.29)$ & $-0.2(0.06)$ & $1.0(1.31)$ & $-0.3(0.04)$ & $-0.2(-0.30,-0.02)$ & 0.22 & 0.0286 \\
\hline Language-thought disorder & $2.3(0.62)$ & $-0.8(0.07)$ & $2.2(0.66)$ & $-1.0(0.05)$ & $-0.2(-0.35,-0.00)$ & 0.19 & 0.0475 \\
\hline Increased motor activity-energy & $2.9(0.7 \mathrm{I})$ & $-I . I(0.09)$ & $2.9(0.64)$ & $-1.4(0.07)$ & $-0.3(-0.52,-0.09)$ & 0.24 & 0.0052 \\
\hline Elevated mood & $2.6(0.95)$ & $-0.9(0.08)$ & $2.8(0.88)$ & $-1.3(0.06)$ & $-0.4(-0.65,-0.24)$ & 0.39 & $<0.0001$ \\
\hline Sleep & $2.4(0.90)$ & $-0.8(0.09)$ & $2.4(0.89)$ & $-1.2(0.06)$ & $-0.4(-0.64,-0.23)$ & 0.32 & $<0.0001$ \\
\hline Sexual interest & $1.6(1.18)$ & $-0.6(0.08)$ & $1.6(1.16)$ & $-0.8(0.06)$ & $-0.3(-0.44,-0.07)$ & 0.24 & 0.0076 \\
\hline Content & $4.4(2.24)$ & $-1.5(0.16)$ & $4.3(2.11)$ & $-2.2(0.11)$ & $-0.6(-1.0 \mathrm{I},-0.27)$ & 0.26 & 0.0008 \\
\hline Speech & $4.4(1.55)$ & $-1.8(0.15)$ & $4.4(1.57)$ & $-2.3(0.11)$ & $-0.5(-0.82,-0.11)$ & 0.28 & 0.0101 \\
\hline
\end{tabular}

Note: ${ }^{P}$ Patients with baseline YMRS hostility-related item scores $\geq 2$.

Abbreviations: $\mathrm{Cl}$, confidence interval; ES, effect size; LS, least squares; LSMD, LS mean difference; MMRM, mixed-effects model for repeated measures; SD, standard deviation; SE, standard error of the mean; YMRS, Young Mania Rating Scale. 
hostility item scores in these patients (irritability: $\mathrm{ES}=0.32$, $P=0.001$; disruptive-aggressive behavior: $\mathrm{ES}=0.45$, $P<0.0001$ ) (Figure 1 and Table 2). For the remaining YMRS items, significantly larger changes from baseline to day 21 were observed for asenapine compared to placebo, with ES ranging from 0.19 to 0.39 (Table 2). Similar results were observed on the YMRS total and item scores in the PANSS hostility subgroup (Table S1).

Following adjustment for the sum of the other nine YMRS items, the difference in change from baseline remained statistically significant in favor of asenapine versus placebo on the disruptive-aggressive behavior score (LSMD [95\% CI] $=-0.4[-0.62,-0.09], P=0.0081)$ and the sum of the disruptive-aggressive behavior and irritability scores $(-0.6[-1.06,-0.09], P=0.0203)$ (Figure 1$)$. Differences between asenapine and placebo did not meet statistical significance on the irritability item score $(-0.2$ $[-0.48,0.07], P=0.1412)$ following adjustment for other mania symptoms.

\section{PANSS hostility and PANSS-EC agitation subgroups}

Baseline PANSS hostility item scores were similar between treatment groups (Table 1). In the PANSS hostility subgroup, the difference between asenapine and placebo was statistically significant on the PANSS hostility item score at day 21 (LSMD $[95 \% \mathrm{CI}]=-0.2[-0.44,-0.04] ; P=0.0181$ ) (Figure 2A). A significant difference in favor of asenapine was also evident at day $7(-0.3[-0.47,-0.12] ; P=0.0010)$ but was lost at day $14(-0.1[-0.39,0.15] ; P=0.3946)$. After adjustment for improvements in PANSS positive symptoms, significant changes from baseline in favor of asenapine were observed at day $7(-0.2[-0.37,-0.04] ; P=0.0151)$ but not at day $21(-0.1[-0.32,0.05], P=0.1582)$. Similarly, after adjustment for improvements in PANSS positive symptoms and sedation, a statistically significant difference was noted for asenapine versus placebo at day $7(-0.2[-0.38,-0.04]$; $P=0.0171)$ but not at day $21(-0.1[-0.33,0.06]), P=0.1634)$. Similar results on both the unadjusted and adjusted PANSS hostility item scores were observed in the YMRS hostility subgroup (data not shown).

Baseline PANSS-EC total scores in the PANSS-EC agitation subgroup were similar between treatment groups (Table 1). Statistically significant changes from baseline to day 21 in favor of asenapine versus placebo were observed on the PANSS-EC total score in agitated patients $(\mathrm{ES}=0.25$, $P<0.01$ ) (Figure 2B). PANSS-EC response (ie, $\geq 40 \%$ improvement from baseline at day 21) was achieved by 24/115 (20.9\%) placebo- and 75/232 (32.3\%) asenapinetreated patients in the PANSS-EC agitation subgroup

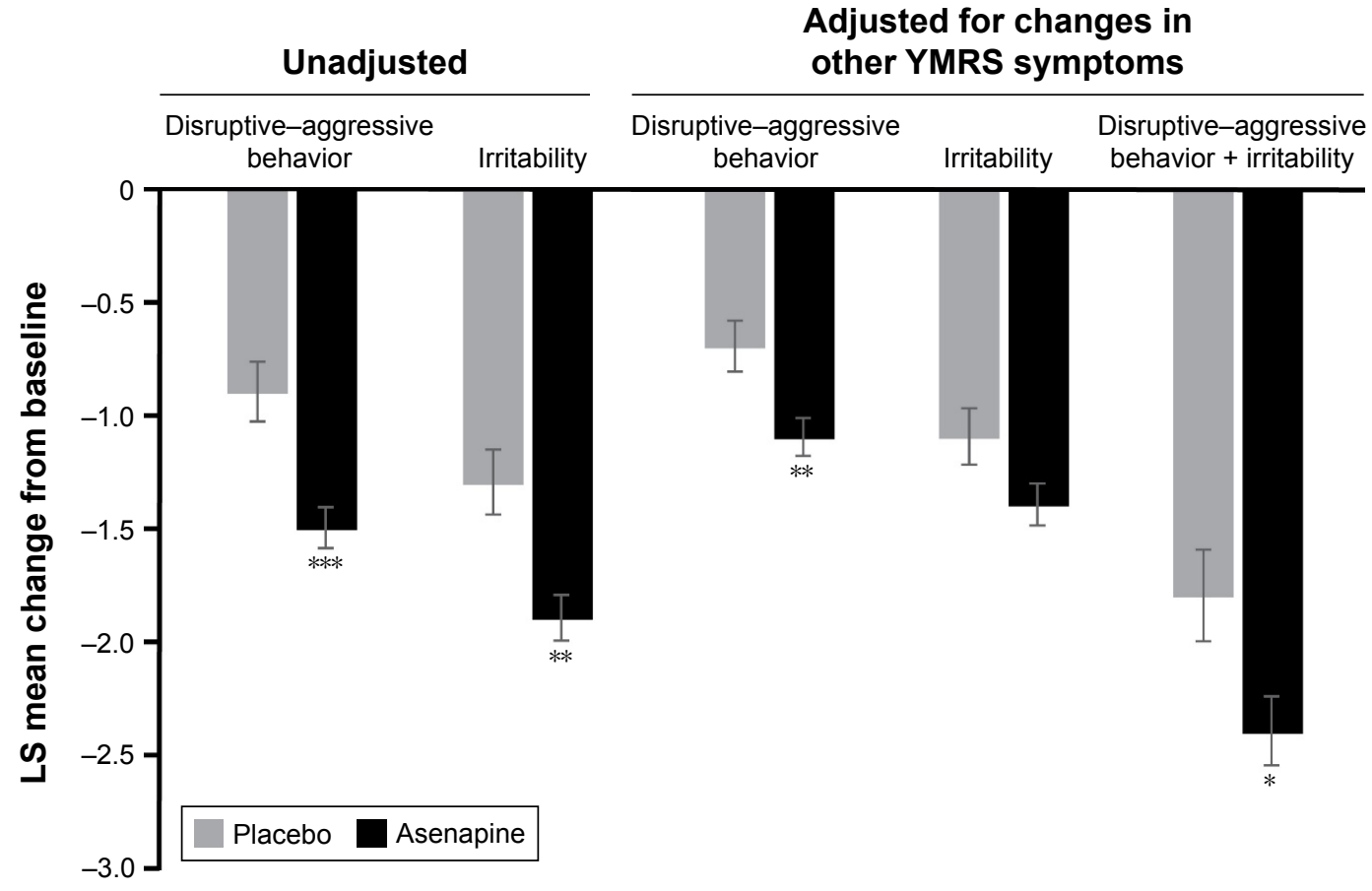

Figure I Changes from baseline to day 21 in YMRS hostility-related items in the YMRS hostility subgroup.

Notes: Patients in this subgroup had baseline YMRS hostility-related item scores $\geq 2$. $* P<0.05 ; * * P<0.01$, *** $P<0.00$ I. Error bars indicate the standard error of the mean. Abbreviations: LS, least squares; YMRS, Young Mania Rating Scale. 

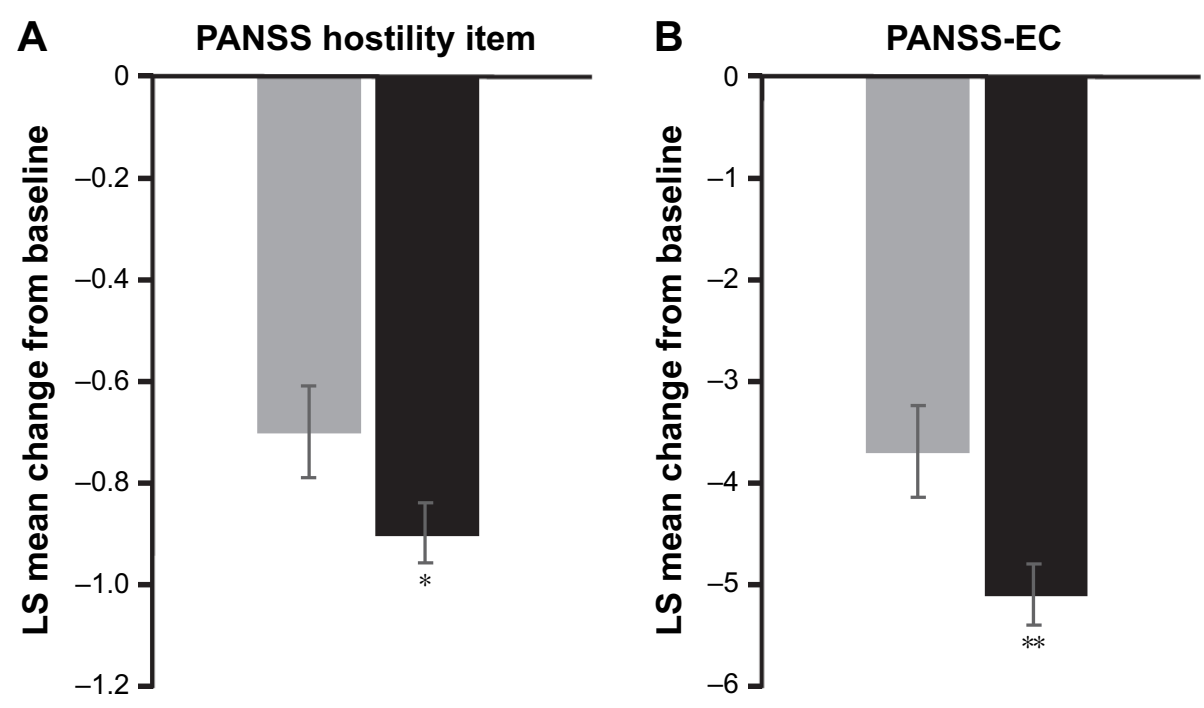

Placebo

Asenapine

Figure 2 Changes from baseline to day 21 in PANSS-derived scores (MMRM).

Notes: (A) PANSS hostility item (P7) score changes in the PANSS hostility subgroup (patients with baseline hostility item scores $\geq 2$ ). (B) PANSS-EC total score changes in the PANSS-EC agitation subgroup (patients with PANSS-EC total score $\geq 14$ and score $\geq 4$ on $\geq 1$ individual item at baseline). Error bars indicate the standard error of the mean. $* P<0.05 ; * * P<0.01$.

Abbreviations: LS, least squares; MMRM, mixed-effects model for repeated measures; PANSS, Positive and Negative Syndrome Scale; PANSS-EC, PANSS-excited component.

(odds ratio $[95 \% \mathrm{CI}]=1.64[0.91,2.94], P=0.099$; number needed to treat $[\mathrm{NNT}]=9[95 \%$ CI 5,53$])$.

\section{Discussion}

Asenapine produced an antihostility effect in patients with bipolar I disorder and symptoms of hostility, as determined by multiple measures of hostility and agitation. Compared with placebo-treated patients, asenapine-treated patients had significantly greater improvements on YMRS hostility-related items (ie, irritability and disruptive-aggressive behavior), as well as the PANSS hostility item at day 21. Significantly greater improvement in overall mania symptoms, as measured by YMRS total score and the YMRS items, was also seen in asenapine- versus placebo-treated patients. Improvements in the YMRS disruptive-aggressive behavior item and the sum of the disruptive-aggressive behavior and irritability items remained significant in favor of asenapine even after controlling for improvements in other mania symptoms, suggesting that improvements in these symptom domains were independent of general improvements in mania. Additionally, significantly greater improvements in symptoms of agitation, as measured by changes from baseline in PANSS-EC total score, were observed with asenapine versus placebo. Taken together, these post hoc analyses using different measures of agitation and hostility suggest that asenapine may be an effective treatment for these symptoms in patients experiencing an acute manic or mixed episode of bipolar I disorder.
Interestingly, although changes in the PANSS hostility item significantly differed between asenapine- and placebotreated patients with high baseline hostility scores at day 21 , these changes were not significantly different after adjusting for positive symptoms and sedation. In a study of the validity of individual PANSS items, hostility displayed overlap between other PANSS items (eg, uncooperativeness, hallucinatory behavior), suggesting that it may be poorly differentiated; additional significant overlap within the individual scores (ie, -3 to +3 ) suggests that this item may also be inherently difficult for raters. ${ }^{24}$ Furthermore, as the component studies were not designed to assess hostility, it is also possible that symptoms of hostility may have been overlooked, causing scores on that particular item to be less precise.

Our findings of antiagitation effects for asenapine are supported by a previous double-blind study of asenapine in acutely agitated patients with any diagnosis, which showed significantly greater improvements in PANSS-EC at 2 hours for patients treated with a single dose of asenapine $(10 \mathrm{mg})$ compared with patients treated with placebo $(P<0.001) .{ }^{18}$ In that study, the effect of asenapine was rapid, with statistically significant differences versus placebo seen as early as 15 minutes after asenapine administration $(P<0.002)$. Efficacy for asenapine was also supported by significantly greater PANSS-EC response rates $(\geq 40 \%$ reduction in PANSS-EC) for asenapine-treated patients (78\%) than for 
placebo-treated patients $(33 \%)(P<0.0001 ; \mathrm{NNT}=3) .{ }^{18}$ Although the current post hoc analyses were not designed to investigate the rapid effect of asenapine, our results extend the findings from acutely agitated patients and suggest that BID doses of asenapine over 3 weeks of treatment were effective against symptoms of agitation and hostility in patients with bipolar I disorder. A small, prospective, naturalistic study conducted on an inpatient psychiatric unit was also supportive of the effects of asenapine on agitation and aggression. In that study, treatment with asenapine (three patients with schizophrenia and two patients with bipolar disorder) was associated with a significant reduction in total aggression as measured by the Modified Overt Aggression Scale, including physical aggression, compared to treatment that did not include asenapine. ${ }^{25}$

Our findings are consistent with findings from previous studies of atypical antipsychotics used to treat patients with bipolar disorder and symptoms of hostility or agitation. Aripiprazole and quetiapine monotherapy demonstrated antihostility effects in patients with bipolar disorder via improvement on YMRS hostility-related items after 3 and 12 weeks of treatment, respectively. ${ }^{13,26}$ Additionally, olanzapine, quetiapine, loxapine, and aripiprazole have demonstrated efficacy in the rapid treatment of agitation in acutely agitated patients with bipolar mania, as measured by improvements in the PANSS-EC subscale. ${ }^{10,12,14,27-29}$

Effective management of hostility and agitation in psychiatric patients includes the short-term relief of acute symptoms, as well as the prevention of acute episodes of hostile/aggressive behavior in the long term. ${ }^{9}$ Although oral antipsychotics are a comprehensive, long-term treatment option for bipolar disorder and symptoms of agitation and hostility, there is concern that orally administered agents may not have a sufficiently rapid onset of action to treat acute or emergent behaviors. Asenapine, which is approved for acute and maintenance treatment of bipolar disorder, may be unique in offering both a rapidly absorbed, noninvasive method of administration and acute and longer term antihostility effects. Therefore, asenapine is a comprehensive treatment option that may be preferable to other antipsychotics in patients with bipolar disorder and symptoms of hostility and agitation; however, direct head-to-head comparisons with other antipsychotics would be desirable.

The pharmacological profile of asenapine may underlie its neurochemical effects and its efficacy on hostile and aggressive behavior. It has been proposed that greater antagonist affinity at $\mathrm{D}_{4}$ receptors compared to $\mathrm{D}_{2}$ receptors (ie, $\mathrm{D}_{4} / \mathrm{D}_{2}$ affinity ratio $>1$ ) may confer antiaggression effects and may explain why some medications display greater efficacy for these behaviors. ${ }^{30}$ Asenapine has slightly higher binding affinity with $\mathrm{D}_{4}$ receptors than $\mathrm{D}_{2}$ receptors, ${ }^{31}$ and this $\mathrm{D}_{4} / \mathrm{D}_{2}$ ratio may therefore be involved in the antiaggression and antihostility effects seen in these analyses. ${ }^{18,30}$ In addition to dopamine receptor affinity, the high affinity of asenapine for $5-\mathrm{HT}_{2 \mathrm{~A}}$ receptors may play a role in antiaggressive efficacy. ${ }^{31}$ Previous studies have shown that antagonism at 5- $\mathrm{HT}_{2 \mathrm{~A}}$ receptors reduces aggressive behavior in preclinical models ${ }^{32}$ as well as in patients with neuropsychiatric disorders. ${ }^{33}$

Limitations of these analyses include their post hoc nature, pooled design, and lack of active comparator. Due to the short duration of the pooled studies, the full effect of asenapine may not have been established. As is common in descriptive analyses, $P$-values were not adjusted for multiple comparisons. Patients in the current study were not specifically selected for a history of hostile/aggressive behavior; hostility in these patients was largely determined by verbal expressions of hostility and not physical assault. Therefore, these results may not be generalizable to other populations, including overtly aggressive patients. Because doses were pooled, it is not possible to identify a dose-dependent effect of asenapine on hostility.

\section{Conclusion}

Treatment with asenapine was superior to placebo in reducing hostility symptoms and agitation in patients with bipolar I disorder, suggesting that asenapine may be an effective and noninvasive treatment for hostility and agitation in this patient population. Improvements on the YMRS hostility factor at endpoint with asenapine treatment were independent of improvements on the remaining YMRS items, indicating that asenapine may have a direct effect on hostility.

\section{Acknowledgments}

Xiao $\mathrm{Wu}, \mathrm{PhD}$, of Allergan, contributed statistical assistance to the manuscript. Writing assistance and editorial support for this manuscript were provided by Jennifer Fetting, $\mathrm{PhD}$, of Prescott Medical Communications Group, Chicago, IL, USA, a contractor of Allergan.

This study was supported by funding from Forest Research Institute, Inc, an Allergan affiliate, Jersey City, NJ, USA. Forest Laboratories, LLC, an Allergan affiliate, was involved in the study design, collection (via contracted clinical investigator sites), analysis, and interpretation of data and the decision to present these results.

\section{Author contributions}

Leslie Citrome, Ronald Landbloom, and Willie Earley contributed to concept/design of these analyses and data analysis/interpretation. Cheng-Tao Chang was involved in 
the statistical analysis and interpretation of data. All authors contributed toward data analysis, drafting and critically revising the paper, and agree to be accountable for all aspects of the work.

\section{Disclosure}

In the past 48 months, Leslie Citrome has engaged in collaborative research with or received consulting or speaking fees from Acadia, Alexza, Alkermes, Allergan, AstraZeneca, Avanir, Boehringer Ingelheim, Bristol-Myers Squibb, Eli Lilly, Forum, Genentech, Intra-Cellular Therapeutics, Janssen, Jazz, Lundbeck, Merck, Medivation, Mylan, Neurocrine, Novartis, Noven, Otsuka, Pfizer, Reckitt Benckiser, Reviva, Shire, Sunovion, Takeda, Teva, Valeant, and Vanda. Willie Earley and Cheng-Tao Chang are employees of Allergan. Ronald Landbloom is an employee of Merck. The authors report no other conflicts of interest in this work.

\section{References}

1. Volavka J. Violence in schizophrenia and bipolar disorder. Psychiatr Danub. 2013;25(1):24-33.

2. Corrigan PW, Watson AC. Findings from the National Comorbidity Survey on the frequency of violent behavior in individuals with psychiatric disorders. Psychiatry Res. 2005;136(2-3):153-162.

3. Pulay AJ, Dawson DA, Hasin DS, et al. Violent behavior and DSM-IV psychiatric disorders: results from the national epidemiologic survey on alcohol and related conditions. J Clin Psychiatry. 2008;69(1):12-22.

4. Latalova K. Bipolar disorder and aggression. Int J Clin Pract. 2009; 63(6):889-899.

5. Garno JL, Gunawardane N, Goldberg JF. Predictors of trait aggression in bipolar disorder. Bipolar Disord. 2008;10(2):285-292.

6. Barlow K, Grenyer B, Ilkiw-Lavalle O. Prevalence and precipitants of aggression in psychiatric inpatient units. Aust $N Z J$ Psychiatry. 2000;34(6):967-974.

7. Binder RL, McNiel DE. Effects of diagnosis and context on dangerousness. Am J Psychiatry. 1988;145(6):728-732.

8. Nolan KA, Volavka J, Czobor P, et al. Aggression and psychopathology in treatment-resistant inpatients with schizophrenia and schizoaffective disorder. J Psychiatr Res. 2005;39(1):109-115.

9. Citrome L, Volavka J. The psychopharmacology of violence: making sensible decisions. CNS Spectr. 2014;19(5):411-418.

10. Citrome L. Inhaled loxapine for agitation revisited: focus on effect sizes from 2 Phase III randomised controlled trials in persons with schizophrenia or bipolar disorder. Int J Clin Pract. 2012;66(3):318-325.

11. Volavka J, Czobor P, Citrome L, Van Dorn RA. Effectiveness of antipsychotic drugs against hostility in patients with schizophrenia in the Clinical Antipsychotic Trials of Intervention Effectiveness (CATIE) study. CNS Spectr. 2014;19(5):374-381.

12. Zimbroff DL, Marcus RN, Manos G, et al. Management of acute agitation in patients with bipolar disorder: efficacy and safety of intramuscular aripiprazole. J Clin Psychopharmacol. 2007;27(2):171-176.

13. Ostacher MJ, Suppes T, Swann AC, et al. Patterns of response to aripiprazole, lithium, haloperidol, and placebo across factor scores of mania. Int J Bipolar Disord. 2015;3:11.

14. Kwentus J, Riesenberg RA, Marandi M, et al. Rapid acute treatment of agitation in patients with bipolar I disorder: a multicenter, randomized, placebo-controlled clinical trial with inhaled loxapine. Bipolar Disord. 2012;14(1):31-40.
15. McIntyre RS, Cohen M, Zhao J, Alphs L, Macek TA, Panagides J. A 3-week, randomized, placebo-controlled trial of asenapine in the treatment of acute mania in bipolar mania and mixed states. Bipolar Disord. 2009;11(7):673-686.

16. McIntyre RS, Cohen M, Zhao J, Alphs L, Macek TA, Panagides J. Asenapine in the treatment of acute mania in bipolar I disorder: a randomized, double-blind, placebo-controlled trial. $J$ Affect Disord. 2010;122(1-2):27-38.

17. Landbloom RL, Mackle M, Wu X, et al. Asenapine: efficacy and safety of 5 and $10 \mathrm{mg}$ bid in a 3-week, randomized, double-blind, placebocontrolled trial in adults with a manic or mixed episode associated with bipolar I disorder. J Affect Disord. 2016;190:103-110.

18. Pratts M, Citrome L, Grant W, Leso L, Opler LA. A single-dose, randomized, double-blind, placebo-controlled trial of sublingual asenapine for acute agitation. Acta Psychiatr Scand. 2014;130(1):61-68.

19. Young RC, Biggs JT, Ziegler VE, Meyer DA. A rating scale for mania: reliability, validity and sensitivity. Br J Psychiatry. 1978;133: 429-435.

20. Kay SR, Fiszbein A, Opler LA. The positive and negative syndrome scale (PANSS) for schizophrenia. Schizophr Bull. 1987;13(2):261-276.

21. American Psychiatric Association. In: Washington DC, editor. Diagnostic and Statistical Manual of Mental Disorders. Fourth ed. St. Louis, MO: American Psychiatric Association; 2000. Text Revision ed.

22. Sheehan DV, Lecrubier Y, Sheehan KH, et al. The Mini-International Neuropsychiatric Interview (M.I.N.I.): the development and validation of a structured diagnostic psychiatric interview for DSM-IV and ICD-10. J Clin Psychiatry. 1998;59(suppl 20):22-33. Quiz 34-57.

23. Montoya A, Valladares A, Lizan L, San L, Escobar R, Paz S. Validation of the Excited Component of the Positive and Negative Syndrome Scale (PANSS-EC) in a naturalistic sample of 278 patients with acute psychosis and agitation in a psychiatric emergency room. Health Qual Life Outcomes. 2011;9:18.

24. Santor DA, Ascher-Svanum H, Lindenmayer J-P, Obenchain RL. Item response analysis of the Positive and Negative Syndrome Scale. BMC Psychiatry. 2007;7(1):66.

25. Amon JS, Johnson SB, El-Mallakh RS. Asenapine for the control of physical aggression: a Prospective Naturalist Pilot Study. Psychopharmacol Bull. 2017;47(1):27-32.

26. Buckley PF, Paulsson B, Brecher M. Treatment of agitation and aggression in bipolar mania: efficacy of quetiapine. J Affect Disord. 2007; 100(suppl 1):S33-S43.

27. Hsu WY, Huang SS, Lee BS, Chiu NY. Comparison of intramuscular olanzapine, orally disintegrating olanzapine tablets, oral risperidone solution, and intramuscular haloperidol in the management of acute agitation in an acute care psychiatric ward in Taiwan. J Clin Psychopharmacol. 2010;30(3):230-234.

28. Meehan K, Zhang F, David S, et al. A double-blind, randomized comparison of the efficacy and safety of intramuscular injections of olanzapine, lorazepam, or placebo in treating acutely agitated patients diagnosed with bipolar mania. J Clin Psychopharmacol. 2001;21(4):389-397.

29. Dundar Y, Greenhalgh J, Richardson M, Dwan K. Pharmacological treatment of acute agitation associated with psychotic and bipolar disorder: a systematic review and meta-analysis. Hum Psychopharmacol Clin Exp. 2016;31(4):268-285.

30. El-Mallakh RS, McKenzie C. The dopamine D4/D2 receptor antagonist affinity ratio as a predictor of anti-aggression medication efficacy. Med Hypotheses. 2013;80(5):530-533.

31. Citrome L. Asenapine review, part I: chemistry, receptor affinity profile, pharmacokinetics and metabolism. Expert Opin Drug Metab Toxicol. 2014;10(6):893-903.

32. Sakaue M, Ago Y, Sowa C, et al. Modulation by 5-HT2A receptors of aggressive behavior in isolated mice. Jpn J Pharmacol. 2002;89(1): 89-92.

33. Takahashi A, Quadros IM, de Almeida RM, Miczek KA. Behavioral and pharmacogenetics of aggressive behavior. Curr Top Behav Neurosci. 2012;12:73-138. 


\section{Supplementary material}

Table SI Change from baseline to day 21 in YMRS total score and individual items in the PANSS hostility subgroup (MMRM)

\begin{tabular}{|c|c|c|c|c|c|c|c|}
\hline & \multicolumn{2}{|c|}{ Placebo $(n=236)$} & \multicolumn{2}{|c|}{ Asenapine $(n=452)$} & \multicolumn{3}{|c|}{ Asenapine vs placebo } \\
\hline & $\begin{array}{l}\text { Baseline } \\
\text { mean (SD) }\end{array}$ & $\begin{array}{l}\text { LS mean } \\
\text { change (SE) }\end{array}$ & $\begin{array}{l}\text { Baseline } \\
\text { mean (SD) }\end{array}$ & $\begin{array}{l}\text { LS mean } \\
\text { change (SE) }\end{array}$ & $\begin{array}{l}\text { LSMD } \\
(95 \% \mathrm{Cl})\end{array}$ & ES & $P$-value \\
\hline YMRS total score & $29.8(6.02)$ & $-10.5(0.79)$ & $29.9(6.08)$ & $-14.6(0.58)$ & $-4.1(-5.97,-2.25)$ & 0.49 & $<0.0001$ \\
\hline \multicolumn{8}{|l|}{ YMRS items } \\
\hline Disruptive-aggressive behavior & $3.1(I .5 \mathrm{I})$ & $-0.7(0.14)$ & $3.1(1.45)$ & $-1.4(0.10)$ & $-0.7(-0.97,-0.34)$ & 0.38 & $<0.0001$ \\
\hline Irritability & $4.0(1.30)$ & $-1.3(0.14)$ & $4.0(1.29)$ & $-1.8(0.10)$ & $-0.5(-0.84,-0.17)$ & 0.32 & 0.0033 \\
\hline Appearance & $\mathrm{I} .2(0.86)$ & $-0.4(0.06)$ & I.2 (0.9I) & $-0.6(0.05)$ & $-0.1(-0.27,0.02)$ & 0.11 & 0.0852 \\
\hline Insight & $0.9(1.29)$ & $-0.2(0.06)$ & $1.0(1.30)$ & $-0.4(0.04)$ & $-0.1(-0.26,0.01)$ & 0.21 & 0.0669 \\
\hline Language-thought disorder & $2.3(0.6 \mathrm{I})$ & $-0.8(0.08)$ & $2.2(0.66)$ & $-I . I(0.06)$ & $-0.2(-0.4 \mathrm{I},-0.05)$ & 0.29 & 0.0109 \\
\hline Increased motor activity - energy & $2.9(0.68)$ & $-I . I(0.10)$ & $2.9(0.65)$ & $-1.4(0.07)$ & $-0.3(-0.55,-0.1 \mathrm{I})$ & 0.32 & 0.0039 \\
\hline Elevated mood & $2.6(0.96)$ & $-0.9(0.09)$ & $2.8(0.88)$ & $-1.4(0.06)$ & $-0.5(-0.68,-0.28)$ & 0.47 & $<0.0001$ \\
\hline Sleep & $2.3(0.91)$ & $-0.9(0.09)$ & $2.4(0.92)$ & $-1.3(0.07)$ & $-0.4(-0.66,-0.23)$ & 0.47 & $<0.0001$ \\
\hline Sexual interest & $1.6(1.20)$ & $-0.6(0.08)$ & $1.6(1.17)$ & $-0.9(0.06)$ & $-0.3(-0.52,-0.13)$ & 0.31 & 0.0013 \\
\hline Content & $4.4(2.27)$ & $-1.7(0.17)$ & $4.4(2.12)$ & $-2.3(0.12)$ & $-0.5(-0.89,-0.12)$ & 0.21 & 0.0103 \\
\hline Speech & $4.5(1.5 \mathrm{I})$ & $-1.9(0.16)$ & $4.4(1.56)$ & $-2.5(0.11)$ & $-0.6(-0.94,-0.21)$ & 0.28 & 0.0020 \\
\hline
\end{tabular}

Note: aPatients with baseline PANSS hostility item scores $\geq 2$.

Abbreviations: Cl, confidence interval; ES, effect size; LS, least squares; LSMD, LS mean difference; MMRM, mixed-effects model for repeated measures; PANSS, Positive and Negative Syndrome Scale; SD, standard deviation; SE, standard error of the mean; YMRS, Young Mania Rating Scale.

\section{Publish your work in this journal}

Neuropsychiatric Disease and Treatment is an international, peerreviewed journal of clinical therapeutics and pharmacology focusing on concise rapid reporting of clinical or pre-clinical studies on a range of neuropsychiatric and neurological disorders. This journal is indexed on PubMed Central, the 'PsycINFO' database and CAS, and is the official journal of The International Neuropsychiatric Association (INA). The manuscript management system is completely online and includes a very quick and fair peer-review system, which is all easy to use. Visit http://www.dovepress.com/testimonials.php to read real quotes from published authors. 